\title{
NANOSPONGES: A REVIEW
}

\section{HIMANGSHU BHOWMIK, D. NAGASAMY VENKATESH*, ANUTTAM KUILA, KAMMARI HARISH KUMAR}

Department of Pharmaceutics, JSS College of Pharmacy, Rockland's, Ooty 643001, JSS Academy of Higher Education and Research (Deemed to be University), Mysuru, India

Email: mailhimangshu12@gmail.com

Received: 29 Jan 2018, Revised and Accepted: 24 May 2018

\section{ABSTRACT}

The recent advance in nanotechnology has lead to the development of targeted drug delivery system. However, targeting a molecule to a particular site using a drug delivery system effectively requires a specialized drug delivery system. The discovery of nanosponge has become a significant step in overcoming certain problems such as drug toxicity, poor bioavailability and release of drug in a predictable fashion as they can accommodate both hydrophilic and hydrophobic drug. Nanosponges exhibit a porous structure in nature which has the unique ability to entrap the drug moieties and offers a merit of desire release. Nanosponges are tiny sponges that can circulate in the body to reach the specific site and binds on the surface to release the drug in a controlled and predictable manner. Nanosponges can be formulated by crosslinking of cyclodextrine with carbonyl or dicarboxylate (Crosslinkers). Nano sponge's technology has been explored widely for the delivery of drugs for oral administration, topical administration, and parental administration. Nanosponges can also serve as an effective carrier for enzyme, proteins, vaccine and antibodies. The present review highlights the method of preparation, characterization and their potential application in drug delivery system.

Keywords: Targeted drug delivery system, Nanosponges, Hydrophilic and Hydrophobic drug, $\beta$-Cyclodextrine

(C) 2018 The Authors. Published by Innovare Academic Sciences Pvt Ltd. This is an open access article under the CC BY license (http://creativecommons.org/licenses/by/4.0/) DOI: http://dx.doi.org/10.22159/ijap.2018v10i4.25026

\section{INTRODUCTION}

To get intended result, targeting drug delivery systems have been an ambition for a prolonged period. In the beginning, Nanosponge drug delivery system appeared only as a topical delivery system, but in the 21st century, Nanosponges can be administered by oral as well as intravenous (IV) route [1].

Nanosponge is a modern category of material and is made up of tiny particles with a narrow cavity of few nanometers. These narrow cavities can be filled with various types of substances. These tiny particles are having a capability due to which it is able to carry both hydrophilic and lipophilic drug substance and can increase the stability of poorly water-soluble drug substance or molecules [2].

The nanosponges are a three-dimensional scaffold (backbone) or network of polyester that are capable of degrading naturally. These polyesters are mixed with a crosslinker in a solution to form Nanosponges. Here, the polyester is generally biodegradable, so it breaks down in the body moderately. Once the scaffold of nanosponges breaks down it releases the drug molecules which is loaded, in a derogatory fashion.

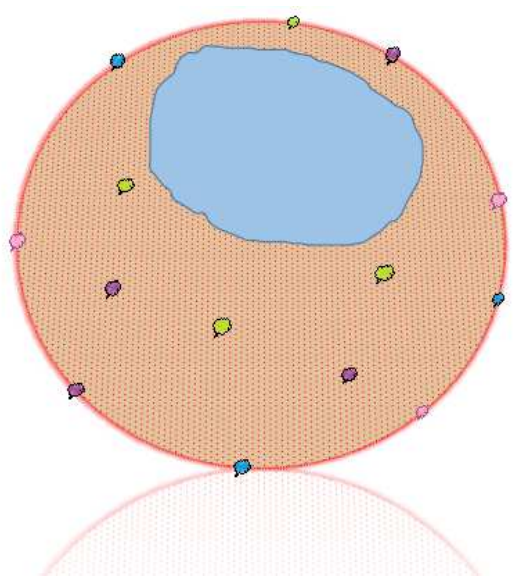

Fig. 1: Structure of a nanosponge showing a cavity for drug loading [3]

\section{Advantages of nanosponges}

$>$ Increase aqueous solubility of the poorly water-soluble drug.

$>$ Nanosponges can release the drug molecules in a predictable fashion.

$>$ Because of their tiny pore size $(0.25 \mu \mathrm{m})$, bacteria cannot penetrate the nanosponges and they act like a self-sterilizer.

$>$ Nanosponges drug delivery system are non-irritating, nonmutagenic and non-toxic.

$>$ Nanosponges help to remove the toxic and venom substance from the body.

$>$ Nanosponges drug delivery system minimize side effect.

$>$ Increase formulation stability and enhance the flexibility of the formulation.

$>$ Reduce dosing frequency.

$>$ Better patient compliance.

$>$ Nanosponges complexes are stable over wide range of $\mathrm{pH}$ (i.e. 111) and a temperature of $130{ }^{\circ} \mathrm{C}[4-6]$.

Disadvantages of nanosponges

$>$ Nanosponges have the capacity of encapsulating small molecules, not suitable for larger molecules.

$>$ Dose dumping may occur at times [7].

Methods of preparation

Solvent method

$>$ Using solvent method, Nano sponges are prepared by mixing polar aprotic solvents like Dimethyl sulfoxide (DMSO), Dimethylformamide (DMF) with the polymer. A crosslinker is then added to this mixture in the ratio of 1:4. The above reaction should be proceeded at temperature $10^{\circ} \mathrm{C}$ to reflux the temperature of the solvent for the time ranging from 1 to $48 \mathrm{~h}$. Once the reaction has completed, the solution is cooled down at room temperature and then obtained a product is added to bi-distilled water. The product is recovered by filtering the product under vacuum and refining by soxhlet extraction with ethanol followed by drying. 
Table 1: Materials used in the preparation of nanosponges

\begin{tabular}{lll}
\hline Polymer & Copolymer & Crosslinker \\
\hline $\begin{array}{l}\text { Hyper cross-linked polystyrenes, } \\
\text { cyclodextrine and its derivatives like }\end{array}$ & $\begin{array}{l}\text { Ethyl cellulose (EC), } \\
\text { polyvinyl alcohol } \\
\text { 2-hydropyclodextrine, }\end{array}$ & $\begin{array}{l}\text { Di-phenyl Carbonate (DPC), diarylcarbonate, diisocyanates, } \\
\text { pyromelliticanhydride, carbonyl diimidazole, 22-bis (acrylamide) acidic } \\
\text { (PVA), }\end{array}$ \\
\end{tabular}

\section{Ultra-sound assisted synthesis}

Polymers are made to react with crosslinkers in a flask without the solvent. The flask is placed in an ultrasound bath which is filled with water and heated up to $90^{\circ} \mathrm{C}$ and the mixture is sonicated for $5 \mathrm{~h}$.
Then the mixture is cooled down to room temperature and then the product is broken into rough pieces. At last, the non-reacting polymer is removed by washing the product with water and refining is done using soxhlet apparatus (ethanol) to obtain nanosponges [10].

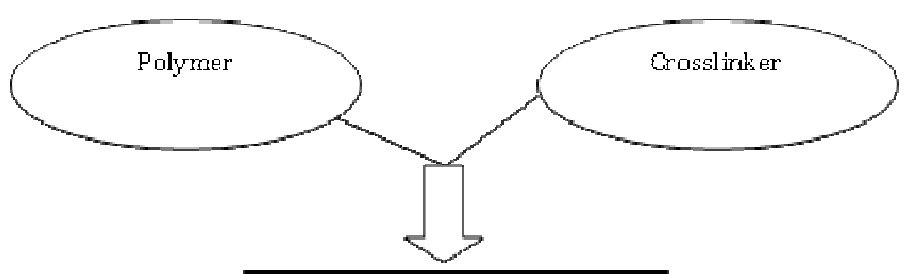

Place the flask in ultra sound

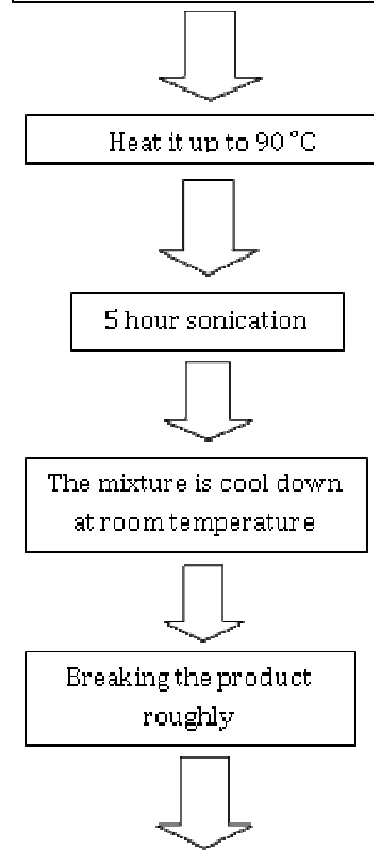

\footnotetext{
Washing of the product with water to remove the non-

reacting polymer and refine it by using soxhlet apparatus
}

Fig. 2: Flow diagram for the preparation of nanosponges by ultrasound-assisted method

\section{Emulsion solvent diffusion method}

In this method, different proportion or amount of ethyl cellulose and polyvinyl alcohol are used to prepare nanosponges. Two phases are used in this method-dispersed and continuous. The dispersed phase consists of ethyl cellulose and the drug, which is then dissolved in $20 \mathrm{ml}$ of dichloromethane and some amount of polyvinyl alcohol (PVA) is added to $150 \mathrm{ml}$ of the continuous phase (aqueous). Then, the mixture is stirred at the speed of $1000 \mathrm{rpm}$ for about $2 \mathrm{~h}$. The product i.e. the nanosponges are collected by filtration. Finally, the product is dried inanoven at a temperature of $400^{\circ} \mathrm{C}[11]$.

\section{Loading of drug into nanosponges}

To obtain the particle size less than $500 \mathrm{~nm}$, nanosponges should be pre-treated. To obtain this range, the nanosponges are dissolved or suspended in water. The suspended nanosponges are sonicated vigorously to prevent the accumulation. The suspension is centrifuged to produce a colloidal fraction. The supernatant is separated and the sample is dried using a freeze dryer.

An aqueous suspension of nanosponges is prepared. An excess amount of drug is added to the suspension and continuously stirred for the certain period of time for the complexation to occur. After the complexation has taken place, the uncomplexed drug is separated from the complexed drug by using centrifugation. The solid crystals of the nanosponges are obtained by using a freeze dryer or by evaporating the solvent.

This Solid Crystal structure of nanosponges has a crucial rule in complexation of the drug. The drug loading capacities of paracrystalline nanosponges is lesser when compared to crystalline nanosponges. The drug loading takes place as a mechanical mixture in weakly crystalline nanosponges [12]. 


\section{Mechanism of drug release from nanosponges}

Since the nanosponges have an open structure (in the surrounding of nanosponges they do not have any continuous membrane), the active substance is added to the vehicle in an encapsulated form. The encapsulated active substance is able to move freely from the particles into the vehicle until the vehicle gets saturated and the equilibrium is obtained. As soon as the product is applied on to the skin, the vehicle containing the active ingredient gets unsaturated causing a disturbance in the equilibrium. Thus, the flow of active substances from nanosponge particles into vehicles starts to epidermis until the vehicle is either absorbed or dried. Even after the retention of the nanosponge particles on the surface of skin i.e. the stratum corneum, the release of active substance continues to skin for a long period of time.

\section{Factors influencing in the formulation of nanosponges}

\section{Nature of polymer}

The polymer used in the preparation of nanosponges can influence its formation and can also affect the pre-formulation. The size of the cavity of a nanosponge should be big enough to entrap a drug molecule of a particular size into it for complexation [13].

\section{Drug}

To be complex with nanosponges, the drug molecules should have some specific characteristics as mentioned below:

- The molecular weight of the drug molecule should be in range ranging from 100-400 Daltons.

- Structure of drug molecule should not consist of more than 5 condensed ring.

- The solubility of thedrug in water should be $<10 \mathrm{mg} / \mathrm{ml}$.

- The melting point of the drug should be $<250{ }^{\circ} \mathrm{C}$.

\section{Temperature}

Changes in the temperature can affect the complexation of drug or nanosponges. Increasing the temperature generally decreases the extent of the stability constant of the drug or the nanosponge complex which may be due to the reduction of interaction forces such as hydrophobic forces and Van der Waal forces of drug/nanosponges with an increase in the temperature [14].

\section{Degree of substitution}

The number, position, and type of the substituent of the parent molecule can affect the ability of complexation of the nanosponges to a greater extent [15].

\section{Method of preparation}

The method of drug loading into the nanosponges can cause a change in the complexation of drug and the nanosponges. Although, the success of a method mainly depends on the nature or the characteristics of the drug and polymer; in some cases, freeze drying has also been known to affect the drug and nanosponge complexation.

\section{Characterization of nanosponges}

The characterization methods for the complexed drug/nanosponges are listed below:

\section{Solubility studies}

Inclusion complexes is a technique by which can determine the solubility and bioavailability of the drug. This technique is the most widely approached technique for analysis of the inclusion complexes of nanosponges. Degree of completion can be known by the plot of phase solubility. Solubility studies are conducted to access the $\mathrm{pH}$ of the drug, solubilization outline and to evaluate the factors affecting drug solubility [16].

\section{Microscopic study}

Microscopic studies of nanosponges/drug can be conducted by using scanning electron microscope and transmission electron microscope. Inclusion complex formation is indicated by the difference in the crystallization state and the product seen under an electron microscope.

\section{Zeta potential determination}

Zeta potential can be defined as the difference of potential between two layers (dispersion medium and immobile layer) of fluid locked up with dispersed particles. Zeta potential is the major key indicator for the stability of the colloidal dispersion. By adding extra electrode on particle size equipment or zeta seizer, the zeta potential can be measured. Higher the value of zeta potential of a colloidal dispersion more is its stability.

\section{Thermodynamical method}

If any changes occur in drug molecules or particles undergoes some changes earlier then the thermal degradation of nanosponges it can be determined by the thermo-chemical method. The changes of drug particles can be melting, evaporation, oxidation and decomposition and polymeric changes. The changes in the drug molecules indicate the formation of a good complex.

\section{Particle size and polydispersity}

Particles size is determined by the process of dynamic light scattering using 90Plus particle size determining software. Dynamic light scattering (DLS) is defined as a technique used to find out the size distribution profile of nanoparticles. At last, the final diameter of the particles and poly-dispersity index (PDI) can be found.

\section{Thin layer chromatography (TLC)}

TLC can be defined as a technique which can be used to separate the non-volatile or evaporative mixture. In this technique, if the Rf value of a particular drug molecule is of an acceptable range then it is helpful in recognizing the formation of a complex between drug and nanosponges.

\section{Infrared spectroscopy}

The interaction between nanosponges and the drug in the solid state can be determined by using infrared spectroscopy. Nanosponge bands can slightly change during formation of complexes. Few guest molecules attached in the complexes which are less than $25 \%$, the drug spectrum can be easily masked by the spectrum of nanosponges. The technique is not appropriate to identify the inclusion complex over the other methods [17].

\section{Loading efficiency}

The loading efficiency of a nanosponge particle can be determined by the estimation of drug loaded into the nanosponge using UV spectrophotometer and high-performance liquid chromatography method for the nanosponges. The loading efficiency of nanosponges can be calculated by using the following equation.

$$
\mathbf{L E}=\frac{\text { Actual drug content in nanosponges }}{\text { Theoretical drug content }} \times 100
$$

\section{Application of nanosponges}

Nanosponges have a wide range of application in the pharmaceutical field, because of its biocompatibility and versatility. In the pharmaceutical industry, nanosponges can be used as an excipient for the formulation of tablets, capsules, granules, pallets, suspensions, solid dispersions and topical dosage forms. Nanosponges can accommodate both lipophilic and hydrophilic drug molecules, basically, those drugs substances which belong to the biopharmaceutical classification system (BCS-class II) as well as the poorly water-soluble drug [18].

\section{Nanosponges for drug delivery}

Nanosponges can carry the water-insoluble drug because of their tiny porous structure. To increase the dissolution rate, solubility and permeability of drug nanosponges complexes play a major role. This is reported that $\beta$-cyclodextrine based nanosponges are three or five times more effective to deliver the drug to the targeted site. Nanosponges are generally solid in nature and can be prepared for oral, parental, topical and inhalation dosage form. For the preparation of tablet, capsule i.e. oral administration the nanosponges complexes are dissolved in a suitable excipient like lubricants, diluents and anti-cracking agent. 
Table 2: List of drug molecules encapsulated with nanosponges

\begin{tabular}{lll}
\hline Category & Nanosponges vehicle & Drugs \\
\hline Anticancer & $\beta$-Cyclodextrine & Paclitaxel Camptothecin \\
Breast cancer & $\beta$-Cyclodextrine & Tamoxifen \\
Inflammation & $\beta$-Cyclodextrine & Resveratrol \\
Brain tumor & Poly (valerolactoneallylvalerolactone) and poly & Temozolamide \\
& (valerolactoneallylvalerolactone -oxepanedione) & 18 \\
Antifungal & Ethyl cellulose Polyvinyl alcohol & 19 \\
& $\beta$-Cyclodextrine & Econazole nitrate \\
Cancer therapy & Sodium alginate & Itraconazole \\
Brain tumor & $\beta$-Cyclodextrine & Antisense \\
Oligonucleotides & Poly L-lysine & Dexamethasone \\
& & Viral infection \\
\hline
\end{tabular}

Nanosponges have several properties that boost the product performance and elegance, controlled release, sustained release, decrease skin irritation, improve solubility and increase product flexibility.

\section{Nanosponges for cancer therapy}

Most challenging works nowadays in the pharmaceutical field is the delivery of anticancer drug because of their low solubility. In one article they claim that nanosponge's complex is three times more effective to reduce the growth of tumor then direct injection. The nanosponge's complex load with a drug and expose a targeting peptide that fastens tightly with a radiation-induced cell upper layer on the tumor receptor. When nanosponges confront the tumor cell they stuck on the surface of tumor cell and start to release the drug molecules. The advantage of targeting drug delivery is to get a more effective therapeutic effect at the same dose and with minimized side effect [21].

\section{Nanosponges for delivery of protein}

To study the encapsulating capacity of $\beta$-cyclodextrin-based nanosponges, bovine serum albumin (BSA) was used as a model protein. Protein solution of bovine serum albumin (BSA) is not stable so they are stored in lyophilized form. Proteins can convert to denatured on lyophilization from its native structure. For the formulation and development of protein, the major drawback is that to maintain its native structure and long-term storage during and after processing. For delivery of the protein like Bovine serum albumin (BSA) with the cyclodextrine based, nanosponges can increase the stability of these proteins. Nanosponges have also been used for immobilization of enzyme, encapsulation of protein, for controlled delivery and stabilization [22].

\section{Role of nanosponges for treatment of fungal infections}

Fungal infections of the skin are one of the dangerous diseases in worldwide [23]. Topical therapy is an attractive choice for the treatment of the coetaneous infections due to various advantages such as targeting of drugs to the direct site of infection and reduction of systemic side effects. Econazole nitrate (imidazole) is an antifungal or pharmaceutical fungicide used topically to cure athlete's foot, ringworm, tineapityriasis versicolor, jock itch and vaginal thrush. The available products of econazole nitrate present in the market are cream, ointment, lotion, and solution. Adsorption of econazole nitrate is not significant when it is applied to the skin and effective therapy; need a high concentration of active agents to be combined. For this reason, econazole nitrate nanosponges were fabricated by emulsion solvent method and these econazole nitrate nanosponges were loaded in a hydrogel as a topical delivery for sustained release of the drug [24-25].

Itraconazole is also an antifungal drug comes under biopharmaceutical classification system class II and that has a dissolution rate limited and poor bioavailability. So the aim of this study was to increase the solubility of the itraconazole, so that can solve the bioavailability problem. In these nanosponges, if used $\beta$-cyclodextrine as cross-linked with carbonate bonds and loaded it with itraconazole than the solubility of itraconazole can be increased.

\section{As absorbent in treating poison in blood}

Nanosponges can remove the dangerous poisonous substance from our blood by absorbing the poison. Instead of using antidotes, if we incorporate nanosponges by injection into blood nanosponges can soak up the toxins. In the bloodstream, the nanosponge looks like a red blood cell, tricks toxins into attacking it, and then absorbs it. The number of toxin molecules each nanosponge can absorb depends on the toxin [26].

\section{CONCLUSION}

Nanosponges have been recognized as drug delivery system to encapsulate or accumulate for both hydrophilic and lipophilic drug by forming a complex. They can effectively deliver the drug in a controlled manner at a target site. Nanosponges can be incorporated into topical preparation such as lotions, cream, ointments etc. And liquid or powder form. The advantage of this technology offers targeting the drug to specific site reduces side effects, improve stability, and improve formulation flexibility and better patient compliance. Nanosponges offer application in other areas such as cosmetics, biomedicine, bioremediation process, agrochemistry, and catalysis etc.

\section{ACKNOWLEDGEMENT}

The author is grateful to the Principal, JSS College of Pharmacy, Ooty for providing the necessary facilities to prepare this review article.

\section{AUTHORS CONTRIBUTIONS}

All the authors have contributed equally

\section{CONFLICT OF INTERESTS}

There is no conflict of interest from all the authors

\section{REFERENCES}

1. Yadav GV, Panchory HP. Nanosponges-a boon to the targeted drug delivery system. J Drug Delivery Ther 2013;3:151-5.

2. Bolmal UB, Manvi FV, Rajkumar K, Palla SS, Paladugu A, Reddy KR. Recent advances in nanosponges as drug delivery system. Int J Pharm Sci Nanotechnol 2013;6:1934-44.

3. Shivani S, Poladi KK. Nanosponges-novel emerging drug delivery system: a review. Int J Pharm Sci Res 2015;6:529.

4. Thakre AR, Gholse YN, Kasliwal RH. Nanosponges: a novel approach of drug delivery system. J Med Pharm Allied Sci 2016;78:103-11.

5. Rita L, Amit T, Chandrashekhar G. Current trends in $\beta$ cyclodextrin based drug delivery systems. Int J Res Ayurveda Pharm 2011;2:1520-6.

6. Ahmed RZ, Patil G, Zaheer Z. Nanosponges-a completely new nano-horizon: pharmaceutical applications and recent advances. Drug Dev Ind Pharm 2013;39:1263-72.

7. Singh D, Soni GC, Prajapati SK. Recent advances in nanosponges as drug delivery system: a review. Eur J Pharm Med Res 2016;3:364-71.

8. Jilsha G, Viswanad V. Nanosponges: a novel approach of drug delivery system. Int J Pharm Sci Rev Res 2013;19:119-23.

9. Shringirishi M, Prajapati SK, Mahor A, Alok S, Yadav P, Verma A. Nanosponges: a potential nanocarrier for novel drug delivery-a review. Asian Pacific J Trop Disease 2014;4:19-26. 
10. Nanosponges: A New Approach for Drug Targetting K. Arshad Ahmed, Khan. Int J Pharm Pharm Res 2016;7:381-96.

11. Sharma R, Pathak K. Nanosponges: Emerging drug delivery system. Pharma Stud; 2010. p. 33-5.

12. Indira B, Bolisetti SS. Nanosponges: a new era in drug delivery. J Pharm Res 2012;5:5293-6.

13. Susmitha, Charanjit, V Manisha Reddy, Naveena, V Ram Mohan Gupta. Nanosponges-a concise review of emerging trends. Int J Pharm Res Biomed Anal 2014;3:1-6.

14. Challa R, Ahuja A, Ali J, Khar RK. Cyclodextrins in drug delivery: an updated review. AAPS PharmSciTech 2005;6:E329-57.

15. Selvamuthukumar S, Anandam S, Krishnamoorthy K, Rajappan M. Nanosponges: a novel class of drug delivery system-review. J Pharm Pharm Sci 2012;15:103-11.

16. Trotta F, Zanetti M, Cavalli R. Cyclodextrin-based nanosponges as drug carriers. Beilstein J Org Chem 2012;8:2091-9.

17. Farooq SA, Saini V. Application of novel drug delivery system in the pharmacotherapy of hyperlipidemia. J Chem Pharm Sci 2013;6:138-46.

18. Trotta F, Dianzani C, Caldera F, Mognetti B, Cavalli R. The application of nanosponges to cancer drug delivery. Expert Opinion Drug Delivery 2014;11:931-41.
19. Gidwani B, Vyas A. A comprehensive review of cyclodextrinbased carriers for delivery of chemotherapeutic cytotoxic anticancer drugs. BioMed Res Int 2015;15:1-15.

20. Vyas A, Saraf S, Saraf S. Cyclodextrin based novel drug delivery systems. J Inclusion Phenom Macrocyclic Chem 2008;62:23-42.

21. Naga SJ, Nissankararao S, Bhimavarapu R, Sravanthi S, Vinusha K. Nanosponges: a versatile drug delivery system. Int J Pharm Life Sci 2013;4:2920-5.

22. Güngör S, Erdal MS, Aksu B. New formulation strategies in topical antifungal therapy. J Cosmet Dermatol Sci Appl 2013;3:56.

23. Trotta F. Cyclodextrin nanosponges and their applications. Cyclodextrins in pharmaceutics, cosmetics, and biomedicine. Current and Future Industrial Applications 2011. p. 323-42.

24. Kaur G, Aggarwal G, Harikumar SL. Nanosponge: New colloidal drug delivery system for topical delivery. Indo Global J Pharm Sci 2015;5:53-7.

25. Renu Kadian. Nanoparticles: a promising drug delivery approach. Asian J Pharm Clin Res 2018;11:30-5.

26. Che-Ming J Hu, Ronnie H Fang, Jonathan Copp, Brian T Luk, Liangfang Zhang. A biomimetic nanosponge that absorbs poreforming toxins. Nat Nanotechnol 2013;8:336-40. 